\title{
Aplicação do formalismo CTP em uma ação com operador pseudo-diferencial
}

\author{
Aplication of the CTP formalism in an action with pseudo-differential operator \\ Leandro Oliveira do Nascimento*1@, Alexsandro B. Corrêa ${ }^{2}$ \\ ${ }^{1}$ Universidade Federal do Pará, Faculdade de Ciências Naturais, Breves, PA, Brasil \\ ${ }^{2}$ Universidade Federal do Pará, Faculdade de Física, Belém, PA, Brasil.
}

\begin{abstract}
Recebido em 18 de Julho de 2019. Revisado em 30 de Setembro de 2019. Aceito em 10 de Outubro de 2019.
O formalismo CTP (closed-time-path) é uma ferramenta matemática para calcular funções de Green em sistemas físicos. A principal vantagem desta técnica é a de que, ao contrário do formalismo de Feynman, o formalismo CTP pode ser aplicado em sistemas fora do equilíbrio. Por outro lado, em física da matéria condensada, alguns modelos não locais no espaço-tempo e de baixa dimensionalidade tem sido bastante discutidos na literatura científica, em particular, na descrição de materiais bidimensionais. Dentre estes, destacamos a Pseudo eletrodinâmica quântica que vem sendo aplicada com sucesso na descrição das interações eletrônicas no grafeno e metais de transição dicalcogenados. Neste trabalho introduzimos o formalismo CTP, usando uma discussão voltada para o cálculo de observáveis físicos. Destacamos o fato de que tal formalismo é muito útil quando o vácuo do passado distante é diferente do vácuo do futuro distante. Após essa breve revisão, fazemos uma aplicação original do formalismo CTP na ação da teoria Proca-Pseudo eletrodinâmica quântica em $(0+1)$ dimensões, calculando as diversas funções de Green relevantes, dentre elas: A função de Green de Feynman; Função de Green Dyson; Função de Green de frequência positiva e; Função de Green de frequência negativa.
\end{abstract}

Palavras-chave: Integrais de Trajetória, Diagramas de Feynman, Modelos não locais.

The CTP (closed time path) formalism is a mathematical tool for calculating Green functions in physical systems. The main advantage of this technique is that, unlike the Feynman's formalism, the CTP formalism can be applied in nonequilibrium systems. On the other hand, in condensed material physics, some nonlocal models in the spacetime and of low dimensionality have been widely discussed in the scientific literature, in particular, in the description of two-dimensional materials. Among these, we highlight the Pseudo quantum electrodynamics that has been successfully applied to describe electronic interactions in graphene and transition metal dichalcogenides. In this work, we introduce the CTP formalism, using a discussion aimed at calculating physical observables. We highlight the fact that this formalism is very useful when the vacuum in the distant past is different from the vacuum in the distant future. Following this brief review, we made an original application of CTP formalism in the action of the Proca-Pseudo quantum electrodynamics theory in $(0+1)$ dimensions, calculating several relevant Green functions, including: Feynman's Green function; Dyson's Green Function; Green function of positive frequency and; Green function of negative frequency.

Keywords: Path Integral, Feynman Diagrams, Nonlocal models.

\section{Introdução}

Em física quântica um dos objetos mais básicos é a chamada amplitude de transição entre dois estados arbitrários, um chamado de estado inicial, representado por $\mid$ in $\rangle$, e outro chamado de estado final, representado por $\mid$ out $\rangle$. Parte desse interesse se justifica pelo fato de que a probabilidade de ocorrer uma transição entre esses estados é nada mais do que o módulo quadrático da amplitude de transição. Existem diversas técnicas para se calcular essa quantidade, por exemplo, cálculo de matriz-S, regra de ouro de Fermi ou métodos variacionais [1]. As propriedades gerais do sistema é o que determinarão a técnica

*Endereço de correspondência: leandrophysics@yahoo.com.br mais adequada, tais como: intensidade das interações, dimensionalidade e simetrias.

No contexto de teoria quântica de campos (TQC), o formalismo de Feynman de integrais de trajetória tem enorme destaque, tanto no cálculo de amplitudes de transição quanto pelo fato de ser útil para o cálculo de funções de Green. Funções de Green, por sua vez, são os objetos básicos da TQC. Estas fornecem informações físicas sobre a massa das partículas, correções quânticas a parâmetros da teoria e potenciais efetivos, como na teoria de perturbação em mecânica quântica. Por outro lado, quando aplicado ao cálculo de um observárvel físico dependente do tempo, o formalismo de Feynman somente é válido se os estados iniciais e finais forem iguais para tempos muito grandes no passado e futuro, respectiva- 
mente. Para evitar esse problema, basta impormos que o vácuo é o mesmo entre os estados envolvidos na amplitude de transição, dessa forma, teremos resultados reais com significado físico. Apesar de algumas complicações introduzidas quando flexibilizamos essa condição, uma vez que a amplitude de transição é adequadamente definida no formalismo CTP, os cálculos das Funções de Green bem como a própria teoria de perturbação são muito parecidas ao caso usual no formalismo de Feynman [2].

Em 1961 Schwinger, em um trabalho seminal intitulado "Brownian motion of a quantum particle" [3], desenvolveu uma generalização do método de integrais de trajetória de Feynman que permitiu abandonar a condição de que os estados inicial e final sejam os mesmos em determinados tempos. Dentro de seu formalismo, também chamado de formalismo CTP, basta conhecer um único estado para sermos capazes de calcular qualquer valor médio. Isso representou também um método para se estudar problemas de valor inicial dentro do formalismo funcional da mínima ação 44. Obviamente, isso é mais próximo ao que conhecemos de mecânica quântica básica, por exemplo, o valor médio do Hamiltoniano $H$ no estado inicial é $\langle$ in $|H|$ in $\rangle$, uma quantidade real definida desde que o operador Hamiltoniano seja Hermitiano, ou seja, $H^{\dagger}=H$ [5]. Esse formalismo, por construção, também pode ser usado em sistemas fora do equilíbrio, pois permite incluir, dentro da própria amplitude de transição, qualquer tipo de condição inicial. Algumas aplicações foram feitas na literatura em modelos locais de TQC [2], enquanto modelos não locais ainda não foram explorados dentro do formalismo CTP.

Modelos de TQC não locais podem surgir através da redução dimensional de um modelo local, introduzindo uma não localidade tanto no espaço quanto no tempo, dependendo da dimensionalidade do sistema físico. Esse procedimento foi elaborado em um artigo original de E. C. Marino [6], o qual contém a dedução da Pseudo eletrodinâmica quântica (PQED), um modelo que tem sido bastante aplicado em física da matéria condensada $[7]$ e com possibilidade de extensão para a área de átomos frios presos em redes ópticas [8]. Teorias não locais são um terreno pouco explorado em TQC, em especial, porque boa parte dos resultados em TQC são obtidos para teorias locais e, em geral, pouco se sabe sobre a interpretação física de tais modelos. Entretanto, com a realização experimental de materiais bidimensionais, podemos afirmar que alguns específicos tipos de não localidade já podem ser interpretados fisicamente como versões reduzidas de teorias locais que descrevem a verdadeira interação entre elétrons no material. Nesse artigo vamos abordar uma aplicação do formalismo CTP a um modelo não local, considerando apenas a evolução temporal do sistema. Esse modelo reproduz o potencial de Yukawa quando definido em $(2+1)$ dimensões 8 .

Para exemplificar o processo de redução dimensional, imagine uma folha de grafeno onde os elétrons estão restritos a se mover no plano, sendo assim, dizemos que são elétrons bidimensionais. O fóton, por outro lado, se propaga para fora da folha, caracterizando este como uma partícula tridimensional. As interações eletrônicas são descritas, portanto, pela redução dimensional dos fótons para o plano, gerando assim a não localidade da PQED. Essa não localidade é descrita por um operador de D'Alembert com uma raíz quadrada. É possível mostrar que na PQED a interação entre os elétrons na folha do grafeno é a usual interação de Coulomb $V(r)=e^{2} / 4 \pi r$, sendo que $e$ é a carga elétrica do elétron e $r$ a distância entre os elétrons. Isso ocorre porque a PQED é versão reduzida (definida no plano) da eletrodinâmica quântica (definida no espaço tridimensional). Uma generalização desse resultado foi feito a fim de obter um potencial de Yukawa no plano $\propto e^{-\mu r} / r$, sendo que $\mu$ é um comprimento de interação 8]. Todas essas aplicações são para sistemas em equilíbrio no formalismo de Feynman, aplicações dentro do contexto do formalismo CTP [9] em modelos não locais não foram realizadas até agora.

O artigo está organizado da seguinte maneira: Na seção II discutimos o formalismo CTP fornecendo definições que podem ser generalizadas para qualquer modelo; na seção III introduzimos o modelo não local e calculamos a sua função de Green de dois pontos (propagador); na seção IV incluimos uma interação quártica para simular um oscilador harmônico quântico anarmônico, e calculamos a correção quântica para a energia de vácuo; ao final do artigo incluimos dois apêndices, sendo que no primeiro calculamos todas as funções de Green relevantes para o formalismo CTP no caso de um oscilador harmônico quântico e no segundo utilizamos esse resultado para calcular a conhecida correção quântica a energia de vácuo de um oscilador com interação quártica.

\section{O formalismo CTP}

Nesta seção nós revisamos tanto o formalismo de Feynman quanto o formalismo CTP com o objetivo de explicar o cálculo de observáveis físicos $\mathcal{O}(t)$, dependentes do tempo, dentro do formalismo de integrais de trajetória. Esses observáveis podem ser a energia do sistema, corrente elétrica gerada por um campo elétrico externo ou a magnetização, para exemplificar alguns poucos. Em seguida, discutimos o caso do oscilador harmônico com uma frequência $\omega(t)$ dependente do tempo, representando um caso de aplicabilidade do formalismo CTP.

A amplitude de transição entre dois estados, no formalismo de Feynman, é dada por

$$
\left.Z=\left\langle\text { in }, t_{i}\right| \text { out }, t_{f}\right\rangle=\int D q(t) e^{-\frac{i}{\hbar} S[q(t)]}
$$

sendo que

$$
S[q(t)]=\int_{t_{i}}^{t_{f}} d t L[q(t)]
$$

é a ação do sistema, $L[q(t)]$ é a Lagrangeana do modelo, $t_{i}$ e $t_{f}$ são os tempos inicial e final, respectivamente, 
$\hbar=h / 2 \pi$ é a constante de Planck divida por $2 \pi$ e $q(t)$ é a variável generalizada do problema em questão. Vamos considerar $\hbar=1$ por simplicidade. A Eq. (1) representa uma integral sobre diferentes trajetórias $q(t)$, notando que a principal contribuição vem da trajetória clássica. Dessa forma, esse formalismo nos permite uma maior conexão conceitual entre a mecânica quântica e a mecânica clássica.

Vamos definir o valor esperado de um observável físico $\mathcal{O}(t)=\mathcal{O}^{\dagger}(t)$. Para fazer isso, nós incluimos uma fonte $J(t)$ na Eq. (1), assim,

$$
Z[J]=\int D q e^{-i \int_{t_{i}}^{t_{f}} d t L[q(t), \dot{q}(t)]+i J(t) \mathcal{O}(t)} .
$$

A fonte $J(t)$ deve ser entendida como algum acoplamento da variável (ou campo) com alguma perturbação externa. Por exemplo, ao aplicarmos um campo elétrico externo em um material, nós podemos medir a sua condutividade elétrica. Segue da Eq. (3) que nosso valor esperado é dado por

$$
\left\langle\text { in, } t_{i}|\mathcal{O}(t)| \text { out }, t_{f}\right\rangle=\left.\frac{\delta Z[J]}{\delta J(t)}\right|_{J=0} .
$$

A Eq. (4) é uma simples e elegante solução para se calcular a evolução temporal de qualquer observável dentro do formalismo in-out. Note que, em geral, nós esperamos que $\mid$ in,$\left.t_{i}\right\rangle \neq \mid$ out,$\left.t_{f}\right\rangle$. Nesse caso, nós teríamos $\mathcal{O}(t) \neq \mathcal{O}^{\dagger}(t)$, o que contradiz nossa consideração de que $\mathcal{O}(t)$ é um observável físico, pois, nesse caso, este deveria ser Hermitiano [5]. Isso pode ser facilmente remediado, ao considerarmos que $\left(t_{i}, t_{f}\right) \rightarrow(-\infty,+\infty)$ com $\mid$ in, $\left.t_{i}=-\infty\right\rangle=\mid$ out,$\left.t_{f}=+\infty\right\rangle=|0\rangle$, sendo que $|0\rangle$ é o estado de vácuo. Finalmente, nós obtemos o valor esperado $\mathcal{O}(t)$, ou seja,

$$
\langle 0|\mathcal{O}(t)| 0\rangle=\left.\frac{\delta Z[J]}{\delta J(t)}\right|_{J=0} .
$$

Nós concluimos, portanto, que existe uma relação não trivial entre os estados in e out no passado remoto e futuro distante. Generalizações desse resultado para dimensões maiores, como $\mathrm{D}=(3+1)$, típicas de TQC são imediatas [1].

A seguir, nós mostraremos como obter equações de campo dentro do chamado formalismo in-in, também chamado de formalismo CTP [9]. Este formalismo é a ferramente apropriada para se calcular valores esperados em um determinado estado inicial, i.e,

$$
\left\langle\text { in }, t_{i}|\mathcal{O}(t)| \text { in, } t_{i}\right\rangle \text {. }
$$

A vantagem da Eq. (6), em comparação com a Eq. (4), é a de que a primeira fornece um valor real para qualquer tempo $t_{i}$. Para calcularmos o valor esperado na Eq. (6), dentro do formalismo funcional, seria necessário termos uma amplitude de transição, cuja derivada deve fornecer a Eq. (6). A seguir, veremos como isso pode ser feito por uma simples generalização do formalismo de Feynman.
Inicialmente, nós comparamos as Eqs. (1) e (3) para obtermos a amplitude de transição $Z[J]$ na presença de uma fonte. Sendo assim, temos $Z[J]=\left\langle\right.$ in, $\left.t_{i}\right|$ out, $\left.t_{f}\right\rangle_{J}$. Como no formalismo CTP nós temos apenas o conhecimento do estado inicial, ver Eq. (6), então a solução encontrada por Schwinger foi introduzir uma soma sobre todos os diferentes estados $\mid$ out, $\left.t_{f}\right\rangle$ com diferentes fontes $J_{+}$e $J_{-}$. Sendo assim, obtemos 9

$$
\left.\left.Z\left[J_{+}, J_{-}\right] \equiv \sum_{t_{f}}\left\langle\text { in }, t_{i}\right| \text { out }, t_{f}\right\rangle_{J_{+}}\left\langle\text {out }, t_{f}\right| \text { in }, t_{i}\right\rangle_{J_{-}} \text {. }
$$

Note que o primeiro fator multiplicativo do lado direito da Eq. (7) é idêntico a amplitude de transição de Feynman e que o segundo fator multiplicativo pode ser obtido apenas tomando-se o complexo conjugado da amplitude de Feynman. A combinação de ambos os fatores, entretanto, requer a utilização de duas polarizações diferentes, uma positiva que indica uma evolução progressiva do tempo e outra negativa que implica uma evolução regressiva no tempo.

As diferentes polarizações deverão ser atribuidas a todas as quantidades do modelo, incluindo os campos básicos e fontes. Tanto $\mathcal{O}(t)$ quanto $J(t)$ são, portanto, substituidos por matrizes de ordem dois, ou seja, $J(t) \rightarrow$ $\left(J_{+}(t) J_{-}(t)\right)$ e $\mathcal{O}(t) \rightarrow\left(\mathcal{O}_{+}(t) \mathcal{O}_{-}(t)\right)$ na Eq. (1). Dessa forma, nosso funcional gerador é dado por

$$
\begin{aligned}
Z\left[J_{+}, J_{-}\right] & =\int D q_{+} D q_{-} e^{-i S\left[q_{+}\right]+i S\left[q_{-}\right]} \\
& \times e^{\oint_{C} d t\left(J_{+} \mathcal{O}_{+}(t)-J_{-} \mathcal{O}_{-}(t)\right)},
\end{aligned}
$$

sendo que $C$ é uma arbitrária curva fechada no tempo, lembrando que cada polarização contribui apenas no ramo progressivo ou regressivo no tempo. Na próxima seção, veremos como isso se aplica na prática ao cálculo da função de Green de dois pontos. Além disso, segue da Eq. (8) que

$$
\left\langle\text { in, } t_{i}|\mathcal{O}(t)| \text { in, } t_{i}\right\rangle=\left.\frac{\delta Z[J]}{\delta J_{+}(t)}\right|_{J_{+}=J_{-}=0},
$$

o valor esperado real para qualquer tempo.

Note que a Eq. (8) é o nosso ponto de partida para calcular equações de campo no formalismo desenvolvido por Schwinger. Generalizações para dimensões de ordem maior são triviais até este ponto 9 .

Para exemplificar o tipo de situação na qual esse formalismo é útil, vejamos o exemplo de um oscilador harmônico com frequência $\omega(t)$ dependente do tempo, dada por

$$
\omega(t)=\omega_{\text {in }} \Theta(-t)+\omega_{\text {out }} \Theta(t),
$$

sendo que $\Theta(t)=1$ para $t>0$ e $\Theta(t)=0$ para $t<0$ é a função degrau. Além disso, sem perda de generalidade, $\omega_{\text {in }} \neq \omega_{\text {out }}$ são tomados como constantes positivas e diferentes entre si. A Lagrangeana do modelo, com uma massa unitária $m=1$, é

$$
L[q, \dot{q}, t]=\frac{1}{2} \dot{q}^{2}-\frac{1}{2} \omega(t)^{2} q^{2}
$$


o que implica em uma equação de campo dada por

$$
\ddot{q}+\omega(t)^{2} q(t)=0 .
$$

Da Eq. 12 nós encontramos as soluções no passado remoto $(t \rightarrow-\infty)$,

$$
q_{\text {in }}(t)=\frac{1}{\sqrt{2 \omega_{\text {in }}}} e^{i \omega_{\text {in }} t}
$$

e no futuro distante $(t \rightarrow+\infty)$

$$
q_{\text {out }}(t)=\frac{1}{\sqrt{2 \omega_{\text {out }}}} e^{i \omega_{\text {out }} t} .
$$

Obviamente existe uma transição não trivial de estados após o tempo $t=0$. Note que usamos soluções da equação de movimento com determinadas condições de contorno que não afetam nossas conclusões finais. Vejamos como ficam as funções de Green nos dois formalismos de integrais de trajetória.

Vamos considerar o exemplo do caso da função de Green de frequência positiva, ver apêndice A para maiores detalhes. No formalismo in-out, nós teríamos que

$$
\begin{aligned}
& G_{+}^{\text {in }- \text { out }}\left(t, t^{\prime}\right)=\left\langle\operatorname{in}\left|q(t) q\left(t^{\prime}\right)\right| \text { out }\right\rangle \\
& =\frac{1}{2 \sqrt{\omega_{\text {out }} \omega_{\text {in }}}} e^{-i \omega_{\text {in }} t+i \omega_{\text {out }} t^{\prime}} .
\end{aligned}
$$

Por outro lado, no formalismo in-in, temos que

$$
G_{+}^{\mathrm{in}-\mathrm{in}}\left(t, t^{\prime}\right)=\left\langle\operatorname{in}\left|q(t) q\left(t^{\prime}\right)\right| \mathrm{in}\right\rangle=\frac{1}{2 \omega_{\mathrm{in}}} e^{-i \omega_{\mathrm{in}}\left(t-t^{\prime}\right)} .
$$

Por comparação direta, fica claro que apenas a Eq. (16) reproduz corretamente a função de Green de frequência positiva do oscilador harmônico, dada em detalhes na Eq. (12) do apêndice A. Nós concluimos, portanto, que o formalismo CTP é relevante quando o estado de vácuo é modificado no tempo (ou em outra coordenada). Note que, embora o problema possua duas frequências diferentes ao longo do tempo, a função de Green do estado de vácuo para $t<0$ é completamente determinada pela frequência $\omega_{\text {in }}$. O formalismo de Feynman, de acordo com a Eq. (15), não reproduz esse resultado, fornecendo uma combinação de frequência $\omega_{\text {in }}$ e $\omega_{\text {out }}$. Vale ressaltar que no caso $\omega_{\text {in }}=\omega_{\text {out }}$ ambos os formalismos são aplicáveis. Nosso exemplo é inspirado pelo caso no qual a frequência é dada por $\omega(t)^{2} \propto 1+\tanh t$, discutido com detalhes na Ref. [9], no qual as mesmas conclusões são tomadas.

\section{Modelo Não Local}

Nós consideramos a Lagrangeana, de um modelo não local, dada por

$$
L[q(t)]=\frac{1}{2} \partial^{0} q K\left[\partial_{0}\right] \partial_{0} q,
$$

sendo que $q(t)$ é nossa variável generalizada. Além disso, o operador não local $K\left[\partial_{0}\right]$, o qual chamaremos também de kernel, é dado por

$$
K\left[\partial_{0}\right] \equiv \frac{2 \sqrt{\left(-\partial_{0}^{2}-\mu^{2}\right)}}{-\partial_{0}^{2}} \equiv \int \frac{d \omega}{(2 \pi)} e^{-i \omega t} \frac{2 \sqrt{\left(\omega^{2}-\mu^{2}\right)}}{\omega^{2}} .
$$

sendo que $\mu$ é um parâmetro de massa. O modelo na Eq. (17), quando devidamente generalizado para $(2+1)$ dimensões, fornece um potencial de Yukawa no qual $\mu^{-1}$ representa o comprimento de correlação. Além disso, o kernel escrito na Eq. (18) foi deduzido na Ref. 8], através de uma redução dimensional da Proca eletrodinâmica. Por isso, a ação em questão também é chamada de Proca-Pseudo eletrodinâmica quântica. Note que uma generalização, para um campo escalar real em TQC, para dimensões maiores é possível. Nesse caso, a quantização canônica e as diversas funções de Green foram detalhadas na Ref. 10 para $\mu=0$.

Nosso primeiro passo é calcular o propagador do modelo na Eq. (17) no formalismo CTP. Análogo a Eq. (8), nós podemos definir o gerador funcional das funções de Green, ou seja,

$$
\begin{aligned}
Z\left[J_{+}, J_{-}\right] & =\int D q_{+} D q_{-} e^{-i S\left[q_{+}\right]+i S\left[q_{-}\right]} \\
& \times e^{\oint_{C} d t\left(J_{+} q_{+}-J_{-} q_{-}\right)} .
\end{aligned}
$$

Após isso, é conveniente reescrever nossas variáveis como elementos de matrizes. Dessa forma, o campo se torna

$$
q_{a}=\left(\begin{array}{c}
q_{1} \\
q_{2}
\end{array}\right)=\left(\begin{array}{c}
q_{+} \\
q_{-}
\end{array}\right)
$$

e as correntes são

$$
J_{a}=\left(J_{1} J_{2}\right)=\left(J_{+}-J_{-}\right) .
$$

Usando a Eq. 20) na Eq. 21) e Eq. (19), e integrando sobre $q_{a}$ em Eq. (19), nós encontramos

$$
Z\left[J_{+}, J_{-}\right]=\exp \left(-i J_{a} G_{a b}^{-1} J_{b}\right),
$$

sendo que nós usamos que, para $J_{+}=J_{-}=0$, nós temos $Z\left[J_{+}=J_{-}=0\right]=1$, que representa nossa condição de normalização. Além disso, $G_{a b}^{-1}$ é uma matriz quadrada de segunda ordem

$$
G_{a b}^{-1}=\left(\begin{array}{cc}
G_{++} & G_{+-} \\
G_{-+} & G_{--}
\end{array}\right)
$$

que satisfaz a seguinte equação

$$
\begin{aligned}
& \left(\begin{array}{cc}
\partial_{0}^{2} K\left[\partial_{0}\right] & 0 \\
0 & -\partial_{0}^{2} K\left[\partial_{0}\right]
\end{array}\right)\left(\begin{array}{cc}
G_{++} & G_{+-} \\
G_{-+} & G_{--}
\end{array}\right)= \\
& \left(\begin{array}{cc}
-1 & 0 \\
0 & -1
\end{array}\right) .
\end{aligned}
$$

Por outro lado, as funções de Green de dois pontos são dadas por

$$
G_{a b}^{-1}\left(t, t^{\prime}\right)=\left.(-i)^{2} \frac{\delta^{2} Z}{\delta J_{a}(t) \delta J_{b}\left(t^{\prime}\right)}\right|_{J_{b}=J_{a}=0} .
$$


Portanto, segue da Eq. (19) e Eq. (22) que

$$
\begin{gathered}
G_{++}\left(t, t^{\prime}\right)=\left\langle\operatorname{in}\left|\mathrm{T} q(t) q\left(t^{\prime}\right)\right| \mathrm{in}\right\rangle, \\
G_{--}\left(t, t^{\prime}\right)=\left\langle\operatorname{in}\left|\mathrm{T}^{-1} q(t) q\left(t^{\prime}\right)\right| \mathrm{in}\right\rangle, \\
G_{-+}\left(t, t^{\prime}\right)=\left\langle\operatorname{in}\left|q(t) q\left(t^{\prime}\right)\right| \operatorname{in}\right\rangle
\end{gathered}
$$

e

$$
G_{+-}\left(t, t^{\prime}\right)=\left\langle\text { in }\left|q\left(t^{\prime}\right) q(t)\right| \text { in }\right\rangle,
$$

sendo que $\mathrm{T}$ é o operador de ordenação temporal. $\mathrm{T}^{-1}$ representa o inverso do operador de ordenação temporal. Esses resultados podem ser generalizados para dimensões maiores de maneira direta 9 .

Eqs. 26- 29 funcionam para qualquer teoria de campos. Por conta da definição delas, estas devem obedecer 9

$$
G_{++}-G_{-+}-G_{+-}+G_{--}=0 .
$$

Além disso, em termos da prescrição do espaço de momento, nós temos que $G_{++}\left(t, t^{\prime}\right)$ é o propagador de Feynman, $G_{--}\left(t, t^{\prime}\right)$ é o propagador de Dyson, $G_{-+}\left(t, t^{\prime}\right)$ é o propagador de frequência positiva e $G_{+-}\left(t, t^{\prime}\right)$ é o propagador de frequência negativa. Portanto, nós escrevemos esquematicamente que

$$
G_{a b}^{-1}\left(t-t^{\prime}\right)=\left(\begin{array}{ll}
G_{F}\left(t, t^{\prime}\right) & G_{<}\left(t, t^{\prime}\right) \\
G_{>}\left(t, t^{\prime}\right) & G_{D}\left(t, t^{\prime}\right)
\end{array}\right)
$$

e, de maneira ainda mais simplificada, nós temos

(32)

$$
G_{a b}^{-1}=\frac{1}{\left(-\partial_{0}^{2} K\left[\partial_{0}\right]\right)^{*}}=\left.\int_{-\infty}^{+\infty} \frac{d \omega}{(2 \pi)} \frac{e^{-i \omega\left(t-t^{\prime}\right)}}{2 \sqrt{\left(\omega^{2}-\mu^{2}\right)}}\right|_{*},
$$

sendo que ${ }^{*}$ significa que alguma apropriada prescrição deve ser aplicada antes da integral ser resolvida para, assim, obtermos a função de Green desejada. O resultado da Eq. 32 foi obtido na Ref. 8] dentro do formalismo de Feynman.

A raíz quadrada na Eq. 32 pode ser removida por uma simples parametrização em $\xi$, dada por 11

$$
\frac{1}{2 \sqrt{\omega^{2}-\mu^{2}}}=i \int_{-\infty}^{+\infty} \frac{d \xi}{(2 \pi)} \frac{1}{\omega^{2}-\mu^{2}-\xi^{2}} .
$$

Usando a Eq. (33) na Eq. (32), nós encontramos

$$
G_{a b}^{-1}\left(t, t^{\prime}\right)=\int_{-\infty}^{+\infty} \frac{d \xi}{(2 \pi)} G_{\xi}^{0}\left(t, t^{\prime}\right)
$$

sendo que

$$
G_{\xi}^{0}\left(t, t^{\prime}\right)=\left.\int_{-\infty}^{+\infty} \frac{d \omega}{2 \pi} \frac{i e^{-i \omega\left(t-t^{\prime}\right)}}{\left(\omega^{2}-\mu^{2}-\xi^{2}\right)}\right|_{*} .
$$

Note que, em geral, as diferentes prescrições fornecerão diferentes funções $G_{\xi}^{0}\left(t, t^{\prime}\right)$. A Eq. 34 mostra, portanto, que os propagadores do nosso modelo são dados por uma integral sobre os propagadores de osciladores harmônicos com frequências $\pm \sqrt{\xi^{2}+\mu^{2}}$, obtidas pelo cálculo direto do pólo da Eq. (35). Com efeito, $G_{\xi}^{0}\left(t, t^{\prime}\right)$ é exatamente a função de Green de um oscilador harmônico quântico, conforme revisamos no Apêndice A.

\section{Interação Quártica}

Incluindo um termo de interação quártica na Eq. (17), como feito no Apêndice B, temos

$$
L[q(t)]=\frac{1}{2} \partial^{0} q K\left[\partial_{0}\right] \partial_{0} q+\lambda q^{4}
$$

No Apêndice B, fazemos uma discussão sobre o tratamento perturbativo da interação quártica, ou seja, quando $\lambda \ll 1$. Nossa principal motivação, ao propor tal tipo de interação, é o fato de que essa interação é a mais comum em TQC quando se inicia o estudo de algum método para tratarmos de interações, seja perturbativo ou não. Nesta seção, para efeito de complementaridade, faremos o tratamento do caso não perturbativo quando $\lambda$ não é necessariamente pequeno.

Como já discutido anteriormente, a amplitude de transição é dada por

$$
Z=\int D q_{a} e^{-i \oint_{C} d t\left[q_{a} G_{a b} q_{b} / 2+\lambda\left(q_{a} q_{a}\right)^{2}\right]} .
$$

Note que o termo de interação é incluido na Eq. (37). Como $\lambda$ não é necessariamente pequeno, precisamos adotar outra estratégia, em comparação ao Apêndice B, para estudar o efeito das interações. Um método comum em TQC é incluir um campo auxiliar $\Delta_{a b}$ na Eq. (37). Dessa forma, temos

$$
\begin{aligned}
Z & =\int D q_{a} D \Delta_{a b} e^{-i \oint_{C} d t\left[q_{a} G_{a b} q_{b} / 2-q_{a} \Delta_{a b} q_{b}\right]} \\
& \times e^{-i \oint_{C} d t\left[\left(\Delta_{a b}\right)^{2} / 4 \lambda\right]} .
\end{aligned}
$$

A ideia principal desse método é a de que integrando sobre o campo $\Delta_{a b}$ na Eq. (38), com o auxílio da Eq. (6) do apêndice A (suplemento 1), nós retornamos para a Eq. 37). O leitor mais atento perceberá que na verdade existe uma constante gerada durante esse processo, entretanto, como tal constante não possui dinâmica, pois é independente dos campos, ela é irrelevante para o cálculo de funções de Green.

O campo auxiliar é uma matriz $2 \times 2$, dada por

$$
\Delta_{a b}\left(t-t^{\prime}\right)=\left(\begin{array}{cc}
\Delta_{++}\left(t, t^{\prime}\right) & 0 \\
0 & -\Delta_{--}\left(t, t^{\prime}\right)
\end{array}\right),
$$

sendo que $\Delta_{++}\left(t, t^{\prime}\right)=\left\langle q_{+}(t) q_{+}\left(t^{\prime}\right)\right\rangle$ e $\Delta_{--}\left(t, t^{\prime}\right)=$ $\left\langle q_{-}(t) q_{-}\left(t^{\prime}\right)\right\rangle$. Note que esse é o campo auxiliar adequado para o nosso método CTP. Além disso, a Eq. (38) é quadrática na posição $q(t)$, o que nos permite integrar essa variável, fornecendo

$$
\begin{aligned}
Z & =\int D \Delta_{a b} \operatorname{det}\left[G_{a b}-2 \Delta_{a b}\right]^{-1 / 2} \\
& \times e^{-i \oint_{C} d t\left(\Delta_{a b}\right)^{2} / 4 \lambda} .
\end{aligned}
$$

Nesse ponto, a Eq. 40 depende apenas da dinâmica do campo auxiliar. Podemos usar a identidade

$$
\ln \operatorname{det} A=\operatorname{Tr} \ln A
$$


e, assim, reescrevermos a Eq. 40 como

$$
Z=\int D \Delta_{a b} e^{-\oint_{C} d t \mathcal{L}_{\text {eff. }}\left[\Delta_{a b}\right]}
$$

sendo que a ação efetiva do campo auxiliar é

$$
\mathcal{L}_{\text {eff. }}\left[\Delta_{a b}\right]=\frac{1}{2} \operatorname{Tr} \ln \left(G_{a b}-2 \Delta_{a b}\right)+\frac{i\left(\Delta_{a b}\right)^{2}}{4 \lambda} .
$$

Podemos obter a dinâmica do campo clássico, ou seja, a solução da equação de movimento, obtida pelo princípio variacional

$$
\left.\frac{\delta \mathcal{L}_{\text {eff. }}}{\delta \Delta_{a b}}\right|_{\Delta_{a b}=\Delta_{a b}^{\mathrm{cl}}}=0
$$

Usando a Eq. 43, na Eq. 44, temos

$$
\frac{i \Delta_{a b}^{\mathrm{cl}}}{2 \lambda}-\delta_{a b} \operatorname{Tr} \frac{1}{G_{a b}-2 \Delta_{a b}^{\mathrm{cl}}}=0
$$

que representa a equação de movimento do campo auxiliar. A Eq. (45) é também chamada de equação de gap e tem aplicação no caso da teoria BCS para supercondutores. Por essa equação, notamos que as componentes de $\Delta_{a b}$ são iguais, portanto, $\Delta_{++}^{\mathrm{cl}}\left(t, t^{\prime}\right)=\Delta_{--}^{\mathrm{cl}}\left(t, t^{\prime}\right) \equiv$ $\Delta^{\mathrm{cl}}\left(t, t^{\prime}\right)$. O resultado na Eq. 45 também pode ser generalizado para o caso do oscilador harmônico quântico.

Podemos também explorar o limite perturbativo no qual $\lambda \ll 1$. Nesse caso, os cálculos são muito parecidos com o apêndice B. Primeiro calcumos a função de Green em tempos iguais $t=t^{\prime}$. Usando a Eq. (32), temos

$$
G_{F}(t, t)=\int_{-\infty}^{+\infty} \frac{d \omega}{2 \pi} \frac{1}{2 \sqrt{\omega^{2}-\mu^{2}}} .
$$

A Eq. 46 é logaritmicamente divergente para $\omega$ grande. Por isso, essa integral deve ser calculada usando-se algum método de regularização para separar a parte divergente da parte finita. Aqui, vamos adotar a regularização dimensional, na qual a dimensionalidade da integral é usada para regularizar, ou seja, separar a parte divergente da parte finita. Ao final dos cálculos, usaremos $D \rightarrow 1$ para voltar a integral desejada. Dessa forma, temos que calcular a seguinte integral

$$
G_{F}(t, t)=\int_{-\infty}^{+\infty} \frac{d^{D} \omega}{(2 \pi)^{D}} \frac{\left(M^{2}\right)^{(1-D) / 2}}{2 \sqrt{\omega^{2}-\mu^{2}}},
$$

sendo que introduzimos um parâmetro $M$ com dimensionalidade de massa a fim de manter a dimensionalidade correta da integral. Resolvendo a integral em $\omega[1]$, temos

$$
G_{F}(t, t)=\frac{i^{2}}{8 \sqrt{\pi}} \frac{\Gamma[(1-D) / 2]}{\Gamma[1 / 2]}\left(\frac{M^{2}}{\mu^{2}}\right)^{(1-D) / 2} .
$$

Definindo $\epsilon \equiv(1-D) / 2$, temos

$$
G_{F}(t, t)=-\frac{\Gamma[\epsilon]}{8 \pi}\left(\frac{M^{2}}{\mu^{2}}\right)^{\epsilon} .
$$

Finalmente, fazemos $D \rightarrow 1$ na Eq. 49, , o que significa $\epsilon \rightarrow 0$. Para isso, usaremos duas propriedades válidas para $\epsilon \rightarrow 0$, ou seja,

$$
\Gamma[\epsilon]=\frac{1}{\epsilon}+\Gamma^{\prime}[1]+O(\epsilon)
$$

sendo que $\Gamma^{\prime}[1]=-\gamma$ e $\gamma \approx 0.57$ é a constante de Euler. Além disso,

$$
A^{\epsilon}=e^{\epsilon \ln A}=1+\epsilon \ln A+O\left(\epsilon^{2}\right),
$$

sendo $A$ uma constante arbitrária. Usando essas propriedades, a Eq. 49 fica

$$
G_{F}(t, t)=-\frac{1}{4 \pi}\left(\ln \frac{M^{2}}{\mu^{2}}-\gamma\right)-\frac{1}{4 \pi \epsilon} .
$$

Note que a Eq. (52) possui um termo, dado por $-1 / 4 \pi \epsilon$, que diverge quando $\epsilon \rightarrow 0$. Esta representa a função de Green regularizada, na qual a parte finita está separada da parte divergente. Além disso, a escala $M^{2}$ é proporcional a $\mu^{2}$ e podemos fazer a qualquer momento dos cálculos a consideração de que $M^{2}=\mu^{2}$ por simplicidade. Para calcular a correção a energia do estado perturbado, consideraremos a função de Green renormalizada, ou seja, sem a contribuição que vem do pólo. Para isso, usaremos um processo chamado de subtração minimal [1]. Apesar de não explicarmos todos os detalhes desse processo aqui, podemos manter em mente que, após retirar o pólo, temos que a parte finita é dada por

$$
G_{F}^{\text {Ren. }}(t, t)=-\frac{1}{4 \pi}\left(\ln \frac{M^{2}}{\mu^{2}}-\gamma\right) .
$$

Dessa forma, usando a Eq. (5) do suplemento 2 (apêndice B), temos que a contribuição finita para a correção da energia do vácuo é dada por

$$
\Delta E=\frac{3 \lambda}{16 \pi^{2}} \gamma^{2}
$$

Note que $\lambda$ tem dimensão de energia no modelo não local ao contrário do modelo que exploramos no apêndice B.

\section{Discussões}

Integral de trajetória é um método constantemente usado em teoria quântica de campos, como ferramenta para cálculo de funções de Green. Neste trabalho apresentamos dois métodos, o conhecido método de Feynman e o formalismo de Schwinger, chamado de CTP. As duas versões são similares, mas a versão de Schwinger exige o cálculo de mais tipos de funções de Green e pode ser aplicada em sistemas fora do equilíbrio. Discutimos essas diferenças tendo como base o oscilador harmônico com frequência dependente do tempo.

Para exemplificar a aplicação do método CTP, usamos um modelo com operador pseudo-diferencial análogo a pseudo eletrodinâmica quântica [6]. Para simplificação, 
reduzimos a dimensionalidade do sistema, considerando apenas a sua evolução temporal. Essa aproximação significa considerarmos um limite quântico sem efeitos relativísticos. Modelos não locais são potencialmente úteis para aplicações em materiais bidimensionais, como o grafeno, pois descrevem a dinâmica de elétrons confinados.

Recentemente, foi sugerido que supercondutividade fora do equilíbrio poderia ser gerada devido a formação de Pares de Cooper entre elétrons que pertencem a diferentes vales e bandas na rede hexagonal do grafeno. Essa fase pode ser obtida pela aplicação de luz circulamente polarizada no material, visando selecionar determinado elétron de cada vale. Espera-se que esse tipo de sistema apresente temperatura crítica maior do que o caso no equilíbro 12. O formalismo CTP tem grande potencial de aplicabilidade para esse tipo de situação, pois além de descrever sistemas fora do equilíbro ele pode adequadamente incluir o efeito de um campo eleltromagnético externo como uma condição inicial dentro do formalismo funcional 9 .

Esse artigo pode servir como uma introdução aos estudantes do curso de física que pretendem estudar o método de quantização, em teoria quântica de campos, através do método de integrais de trajetória. Damos especial enfoque aos diferentes tipos de funções de Green e como, em linhas gerais, obter representações integrais para essas funções. Uma possível generalização desse trabalho é incluir efeitos de temperatura.

\section{Agradecimentos}

Nós gostaríamos de agradecer ao Prof. Dr. Van Sérgio da Silva Alves por interessantes discussões e sugestões ao texto final. Alexsandro B. Corrêa agradece ao PIBICUFPA (Programa Institucional de Bolsas de Iniciação Científica) pelo financiamento dessa pesquisa.

\section{Referências}

[1] M.O.C. Gomes, Teoria quântica dos campos (EdUSP, São Paulo, 2015).

[2] E.A. Calzetta, Nonequilibrium quantum field theory (Cambridge University Press, Cambridge, 2008).

[3] J. Schwinger, J. Math. Phys 2, 407 (1961).

[4] F. Cooper, arXiv:hep-th/9504073 (1995).

[5] J.J. Sakurai, Modern quantum mechanics (AddisonWesley Publishing Company, California, 1993).

[6] E.C. Marino, Nucl. Phys. B 408, 551 (1993).

[7] E.C. Marino, L.O. Nascimento, V.S. Alves e C. Morais Smith, Phys. Rev. X 5, 011040 (2015).

[8] V.S. Alves, T. Macrì, G.C. Magalhães, E.C. Marino e L.O. Nascimento, Phys. Rev. D 97, (2018).

[9] R.D. Jordan, Phys. Rev. D 33, 444 (1986).

[10] R.P.L.G. do Amaral e E.C. Marino, J. Phys. A: Math. Gen. 25, 5183 (1992).

[11] I.S. Gradshteyn e I.M. Ryzhik, Table of Integrals, Series, and Products (Academic Press, Cambridge, 1980).
[12] O. Hart, G. Goldstein, C. Chamon e C. Castelnovo, arXiv:1810.12309 (2018).

[13] L.D. Landau e E.M. Lifshitz, Quantum mechanics: Non-relativistic theory (Butterworth-Heinemann, Oxford, 1981). 